\title{
Essais
}

Revue interdisciplinaire d'Humanités

Politicité de la littérature et des arts contemporains

\section{Fac-similé du pouvoir politique : Facsímil d'Alejandro Zambra}

Nicolás Folch Maass

\section{OpenEdition}

1 Journals

Édition électronique

URL : http://journals.openedition.org/essais/938

DOI : 10.4000/essais.938

ISSN : 2276-0970

Éditeur

École doctorale Montaigne Humanités

Édition imprimée

Date de publication : 15 septembre 2020

Pagination : $39-51$

ISBN : 979-10-97024-08-6

ISSN : 2417-4211

\section{Référence électronique}

Nicolás Folch Maass, «Fac-similé du pouvoir politique : Facsímil d'Alejandro Zambra », Essais [En ligne], 16 | 2020, mis en ligne le 28 septembre 2020, consulté le 13 octobre 2020. URL : http:// journals.openedition.org/essais/938; DOI : https://doi.org/10.4000/essais.938 


\title{
Fac-similé du pouvoir politique : Facsímil d'Alejandro Zambra
}

\author{
Nicolás Folch Maass
}

Facsimil ${ }^{1}$ est le sixième livre en prose d'Alejandro Zambra, né en 1975. Le travail de ce jeune écrivain chilien se caractérise par une écriture hybride, où se croisent son premier amour, la poésie, et une diversité de types de langage au service du discours et du récit. L'écriture de Zambra ainsi comprise revendique dans Facsímil son caractère expérimental. L'auteur affirme cette liberté dans l'écriture et met au sommet de celle-ci la création poétique. Selon lui, cette liberté réside avant tout dans une rigueur esthétique qui ne (re)connaît pas les frontières préétablies entre les styles. Cette rigueur était déjà à l'œuvre dans son troisième livre, Bonsái, qui inspira ensuite le film ${ }^{2}$ du même nom :

Écrire est comme prendre soin d'un bonsaï, je le pensais alors, je le pense aujourd'hui : écrire c'est tailler les branches jusqu’à rendre visible une forme qui était présente, cachée [...].

Cette image me plaisait et jai commencé à regarder des manuels sur les bonsaïs. Je voulais écrire un livre de poésie avec ce langage. Je me suis déplacé vers la narration et jai écrit un court récit où il se passe plus ou moins ce quill arrive dans Bonsái. ${ }^{3}$

La complexification des moyens discursifs, guidée par l'élagage des contraintes narratives propres au récit, se matérialise de manière flagrante dans Facsimil. La structure narrative est dissoute par l'effacement partiel de la personnalisation énonciatrice classique et de sa linéarité discursive. Facsimil

Alejandro Zambra, Facsimil, Santiago, Hueders, 2014.

2 Jiménez Cristian, Bonsai, coproduction Chili - Argentine - Portugal - France, 2011, 102 min.

3 Alejandro Zambra, propos recueillis par Leila Guerriero, "Alejandro Zambra, el hombre que lee ", El Pais, [en ligne], disponible sur : https://elpais.com/diario/2011/05/28/ babelia/1306541542_850215.html [consulté le 22/08/2017] : "Escribir es como cuidar un bonsái, pensé entonces, pienso ahora: escribir es podar el ramaje hasta hacer visible una forma que ya estaba alli, agazapada [...]. Me gustaba esa imagen y empecé a mirar manuales de bonsái. Quería escribir un libro de poesía con ese lenguaje. Me fui desplazando hacia la narrativa y escribi un relato corto donde sucedia más o menos lo que sucede en Bonsái ». Toutes les traductions de l'article vers le français sont personnelles. 
mime et parodie l'épreuve de sélection universitaire chilienne, offrant un texte dont l'unité se fonde dans la panoplie des modes discursifs du test de communication et langage nommé au Chili "Prueba de aptitud verbal». Le lecteur est dès lors face à un livre qui ne peut se lire autrement qu'en référence aux règles établies dès l'avertissement placé en épigraphe : "Ce livre suit le schéma de l'Épreuve d'Aptitude Verbale en vigueur jusqu'en 1993 ". La structure du livre en chapitres simule les différentes parties de cette épreuve : "I. Terme à exclure ", " II. Plan de rédaction ", " III. Utilisation de connecteurs ", "IV. Élimination de phrases » et "V. Compréhension de lecture " . L'auteur, lui-même soumis à cet exercice à la fin de sa période lycéenne, établit ainsi un lien entre le langage littéraire et sa propre expérience du langage institutionnel à travers ce test à choix multiples. Cet ancrage intertextuel sera essentiel, comme nous le verrons, pour l'effet ironique (intratextuel) à partir de la parodie $^{6}$ (intertextuelle) dont Zambra fait sa principale stratégie narrative.

\section{L'éducation et l'expression structurante du discours dans Facsímil}

L'" Épreuve d'Aptitude Verbale $»^{7}$ faisait partie d'un ensemble d'épreuves nommé "Épreuve d'Aptitude Académique " ("PAA » ${ }^{8}$ en espagnol), adopté au niveau national à partir de 1967 . Son objectif était de faciliter la sélection des futurs étudiants par les universités chiliennes. Cette forme de sélection nationale ou "Système Unique d'Admission " a été remplacée en 2004 par une autre batterie d'épreuves nommées "Épreuve de Sélection Universitaire " $\left(\right.$ (PSU $\left.»^{10}\right)$. Or, ces deux systèmes, s'ils ont le mérite de traduire une volonté de paramétrer de manière uniforme, donc supposément égalitaire, le processus d'admission aux universités, montrent les mêmes défauts. En effet, les études sur la PAA référencées par Carlos Rodríguez Garcés et Victor Castillo Riquelme, et leur comparaison avec celles de la PSU démontrent que ces systèmes d'admission classent les postulants selon leur origine sociale puisque leurs résultats sont étroitement corrélés à la catégorie socio-économique de leur famille ${ }^{11}$.

4 Alejandro Zambra, Facsimil, op. cit., p. 11 : "Este libro se basa en la Prueba de Aptitud Verbal, según su estructura vigente en 1993 ".

5 "I. Término excluido ", "II. Plan de redacción ", "III. Uso de ilativos", "IV. Eliminación de oraciones» et «V. Comprensión de lectura».

6 Linda Hutcheon, "Ironie, satire, parodie : une approche pragmatique de l'ironie », Poétique, $\mathrm{n}^{\circ}$ 46, Paris, Seuil, 1981, p. 140-155.

7 "Prueba de Aptitud Verbal".

8 "Prueba de Aptitud Académica».

9 "Sistema Único de Admisión».

10 "Prueba de Selección Universitaria".

11 Carlos Rodriguez Garcés, Victor Castillo Riquelme, "Nivel de logro PSU: Lo que devela el puntaje corregido en las Pruebas de Admisión Universitaria en Chile ", Propuesta Educativa [en ligne], disponible sur : http://www.scielo.org.ar/scielo.php?script=sci_arttext\&pid=S1995-77852015000200010 [consulté le 08/07/2017]. 
Facsimil reprend la structure de l'épreuve à laquelle l'auteur a été confronté pour introduire des éléments qui questionnent les effets de l'institutionnalisation du langage. Ainsi, le caractère expérimental de sa structure narrative a comme source l'expérience vécue, sans pour autant en faire un récit autobiographique. L'expérimentation littéraire devient plus radicale encore à travers l'expérience de la lecture. Elle met les lecteurs dans la même situation que des lycéens évalués sur leurs aptitudes linguistiques. Les lecteurs sont ainsi confrontés aux enjeux de l'exemplarité langagière grâce à l'interpellation de leurs habitudes culturelles.

Ce roman met en lumière la régulation dans la production collective de valeurs, tant au niveau littéraire qu'au niveau des individus, par un langage dont le caractère thétique est soumis aux règles du marché économique. Ceci est flagrant quand le narrateur se fait payer pour écrire les mémoires d'un mégalomane sans scrupules : "Nous nous sommes mis d'accord sur un tarif par mot, la seule chose qui l'intéressait était que le livre soit gros ${ }^{12}$ avoue-t-il cyniquement. De cette manière, le langage devient dans ce livre un outil de régulation où les valeurs sociales peuvent être inversées au profit d'un certain modèle de société et d'identité nationale. L'exemplarité linguistique s'érige dès lors comme un mode de légitimation de certaines valeurs identitaires pour des individus issus d'un même système éducatif.

\section{La mimésis de la triche et la critique d'une identité figée}

Le premier exercice de ce " livre-test " met en tension un savoir faire - répondre aux questions par l'élimination d'un choix non pertinent - avec la légitimité de la mimésis littéraire :

1. FAC-SIMILÉ
A) copie
B) imitation
C) simulacre
D) essai
E) piège. ${ }^{13}$

L'intitulé ou " mot guide " de l'exercice détermine la pertinence du terme à exclure en même temps qu'il rappelle le titre exact du livre. Ce début soulève des questions sur ce que pourrait être ce livre en particulier et également sur la nature de la littérature en général. Le lecteur est ainsi invité à prendre position par rapport à ses idées préconçues et à ses compétences culturelles dans le champ

12 Alejandro Zambra, Facsimil, op. cit., p. 61 : "Pactamos una tarifa por palabra, lo único que le importaba era que el libro fuera gordo".

13 Ibid., p. 15 : 1. FACSÍMIL A) copia, B) imitación, C) simulacro, D) ensayo, E) trampa. 
littéraire. L'auteur incite au questionnement des valeurs qui sont couramment acceptées par les individus soumis au test. Dans ce sens, la valeur littéraire du livre est mise sous tension par des alternatives contraires aux valeurs classiques d'originalité et d'honnêteté. En outre, l'allusion au piège ("trampa ") ou à la copie ("copia») inverse le principe tacite selon lequel toute épreuve exclut la tricherie. Facsimil opère une inversion de la logique que Pierre Bourdieu observe dans la soumission des individus à un pouvoir quelconque par l'utilisation de ses valeurs symboliques ${ }^{14}$. Dans le cas du Chili, le pouvoir centralisé de l'État est historiquement solide et bien assuré. Ceci produit un marché de valeurs symboliques fortement réglé par les institutions de l'État, dont l'éducation et ses agents font partie, avec la structure familiale en arrière-plan.

Zambra écrit un livre dont le discours fait apparaittre certaines réalisations sociales privées et étatiques contemporaines (littéraire, éducative et familiale) qui surdéterminent le texte. Cette diégèse ${ }^{15}$ met en lumière certaines valeurs et institutions qui font la promotion d'une identité nationale : le mariage, la loi sur le divorce, le double standard du progrès social et humain dans un système éducatif qui renforce la fraude et la soumission, le machisme hérité du milieu familial, les dates et faits historiques, etc. L'aspect formel de l'écriture en tant que copie d'un autre texte destiné à l'évaluation investit dès lors le sens politique de la technocratie dans l'éducation : "Si l'acte créatif ne se limite point à sa dimension critique, c'est bien parce qu'il interpelle et provoque le monde ${ }^{16}$. L'appropriation littéraire de la forme du PAA effectuée depuis la place publique, cette copie formelle devient critique $^{17}$.

Dans sa deuxième partie, intitulée "Plan de rédaction " ("Plan de redacción»), le livre présente une série d'exercices censés porter un ordre rhétorique qu'il faut reconstituer à partir des choix combinatoires proposés :

31. La famille

1. Tu les classes en deux listes: ceux que tu aimes et ceux que tu n'aimes pas.

2. Tu les classes en deux listes : ceux qui ne devraient pas être vivants et ceux qui devraient être morts.

3. Tu les classes selon le degré de confiance quils tinspiraient quand tu étais petit.

4. Pendant un instant tu penses découvrir quelque chose d'important, quelque chose qui depuis des années attendait une conclusion.

5. Tu les classes en deux listes : les vivants et les morts. ${ }^{18}$

14 Voir Pierre Bourdieu, Langage et pouvoir symbolique, Paris, Seuil, 2001 (1991).

15 Gérard Genette, "Nouveau discours du récit ", Discours du récit, Paris, Seuil, 2007 (1983), p. 301.

16 Hans Cova, «L'engagement (artistique) est-il nécessairement critique? ", in Eric Van Essche et al. (éd.), Les formes contemporaines de l'art engagé, Bruxelles, La Lettre volée, 2007, p. 50.

17 Ibid., p. 48-50.

18 Alejandro Zambra, Facsímil, op. cit., p. 28 : 31. Los familiares. 1) Los clasificas en dos listas: los que amas y los que no amas. 2) Los clasificas en dos listas: los que no deberían estar vivos y los que 
Par la détermination formelle d'origine du livre, le discours se caractérise par une tendance aux formules prédicatives. Or, le discours dévoilera une possible narration et la dimension de sa signification, une fois la lecture de l'ensemble achevée. En effet, les parties de ce livre offrent un récit précaire, interrompu et parfois répétitif où le souci d'une quelconque continuité logique ou d'unité du sujet est presque inexistant. De prime abord, ce livre présente des énoncés qui renvoient aux valeurs tissées par la famille et l'éducation. Comme pour le titre et le renversement de l'honnêteté intellectuelle par la valeur de la tricherie, toutes les valeurs sociales sont empreintes de négativité : la réussite de la vie de couple s'exprime dans un divorce réglé par la loi ${ }^{19}$; les jeunes adultes refusent de devenir des parents et souhaitent rester des enfants, en même temps que les parents travaillent et souffrent de manière absurde ${ }^{20}$. Ceci permet la lecture d'une mémoire collective terrée dans le langage et les lieux communs d'une identité imposée. Cette mémoire est récupérée à rebours de la contemporanéité et offre un regard critique sur des valeurs collectives. Un exemple est le lien entre les individus, faussé par le conditionnement de l'histoire personnelle à partir d'un langage institutionnalisé. Ainsi, par exemple, l'exercice " 31. La famille " établit une encyclopédie intime, une classification binaire de relations : les êtres aimés et les non-aimés, ceux qui devraient être vivants ou non, ceux qui méritent la confiance ou non et les réellement vivants et morts. Le choix $n^{\circ} 4$ est la seule solution proposée qui n’offre pas de hiérarchisation. Il souligne une découverte personnelle (" descubres»). Cette découverte importante, qui solderait des années passées dans l'ignorance ou la confusion, n'est toutefois pas dévoilée. Elle demeure secrète, mais reliée à l'aménagement binaire du monde. Toutefois, la problématique relationnelle devient centrale entre une deuxième personne et les autres. Cet exercice illustre l'impact de l'exemplarité de la langue institutionnalisée. Elle assure le maintien de hiérarchies facilement assimilées par la société. L'éducation instrumentalise ainsi le langage et ceci permet d'indexer le rôle des individus à tous les niveaux, y compris la famille, comme dans l'exercice " 31 ».

Les relations entre les individus sont conditionnées sérieusement par le langage, un outil producteur d'une fatalité sociale. Chacun est réduit à un positionnement binaire dans son interaction avec le monde. Il n'est pas question de choisir, mais d'assumer sa place dans la société. Dans l'exercice "35. Nager» ("35. Nadar»), l'adaptabilité sociale qui permet la survie de l'individu demeure confuse, imbriquée entre la mémoire individuelle et collective : " mais tu savais nager, ils n'ont pas eu à t'apprendre "; " ou peut-être

deberian estar muertos. 3) Los clasificas según el grado de confianza que inspiraban en ti cuando niño. 4) Por un momento piensas que descubres algo importante, algo que estaba pendiente hace años. 5) Los clasificas en dos listas: los vivos y los muertos.

19 Ibid., p. 88.

20 Idem. 
oui, ils t'ont appris à l'école [...]. Non pas à nager, mais à bouger les bras et les jambes. Et à retenir ta respiration pendant des heures ${ }^{21}$. L'éducation que le verbe apprendre (" enseñaron ") évoque intervient dans la vie de manière obscure. Elle modifie les souvenirs, les rend assez flous. Ainsi, la soumission sociale à un système de vie se mêle au sentiment d'une responsabilité individuelle. Cette éducation rend possible - comme le suggère l'image de la respiration retenue des heures durant - la stagnation des individus ou une acceptation de la précarité. L'éducation façonne les aptitudes de chacun au profit de la soumission.

\section{La parodie et l'ironie comme stratégies narratives}

La stratégie textuelle de la parodie et de l'ironie permet également le dépassement d'un savoir topique propre aux lecteurs qui ont vécu sous le même système éducatif chilien. Ainsi, par exemple, les alternatives A, B, C, D et $\mathrm{E}$ proposées pour l'exercice «35. Nager » ne laissent en réalité aucun choix, car toutes offrent la même chronologie possible : 1-2-3-4-5. Ainsi, l'agencement de cette parodie d'épreuve montre de manière ironique qu'il n'y a pas vraiment d'autre choix pour le lecteur que d'accepter l'ordre de ce que l'on pourrait appeler une " ironie situationnelle ${ }^{22}:$ "nager renforce les muscles et la mémoire $»^{23}$ dit le narrateur, alors qu'au bord de la piscine il peine à mettre au clair ses souvenirs. Dans ce sens, l'interpellation de la connaissance de codes et des compétences des lecteurs est universelle, car la transgression des règles du QCM est évidente. Ce même recours à un seul choix possible se réitère dans l'exercice qui suit " 36 . Cicatrices » («36. Cicatrices»), où les alternatives $\mathrm{A}, \mathrm{B}, \mathrm{C}, \mathrm{D}$ et $\mathrm{E}$ n'offrent qu'une possibilité de combinaison : 1-2-3-45-6-7-8-9-10-11. Cette répétition de l'ironie comme transgression des règles sous-jacentes permet le renforcement d'une intentionnalité et d'un processus sémantique qui guide le discours. Les lecteurs peuvent ainsi interpréter une cohérence de l'ensemble, malgré un discours scindé selon les modalités stylistiques de chaque partie de l'épreuve.

Le point de vue que présuppose Facsímil est alors peu à peu éclairé par le renforcement de l'ironie. La relation entre l'éducation et la critique des valeurs nationales est peu à peu soulignée grâce à cette stratégie narrative. Ainsi, par exemple, avec la situation d'un étudiant honnête qui ne suit pas le chemin de la tricherie, Zambra montre l'absurdité du rôle social de l'individu par rapport

21 Ibid., p. 30 : "pero sabias nadar, no tuvieron que enseñarte "; " o quizás sí te enseñaron en el colegio [...]. No a nadar pero si a mover los brazos y las piernas. Y a aguantar la respiración durante horas.".

22 Voir Linda Hutcheon, "Ironie, satire, parodie : une approche pragmatique de l'ironie ", art. cit., p. 140-155.

23 Zambra Alejandro, Facsimil, op. cit., p. 30 : "nadar fortalece los músculos y la memoria ". 
à ses convictions : " [...] j’ai dû étudier Pédagogie en Religion, alors que je ne croyais pas tellement en Dieu $»^{24}$. En effet, cette stratégie aiguise le rôle inquisiteur du lecteur face à l'ironie qui traverse l'ensemble du discours. La parodie de l'épreuve facilite le ressenti de l'effet ironique réitéré plusieurs fois dans le texte. La lecture conduit à la reconnaissance d'un (con)texte culturel sousentendu : PAA ou épreuve à choix multiples. Dans ce cadre allégorique de l'institution éducative, l'ironie apparait pour offrir le revers des valeurs identitaires espérées. Par exemple, avec l'exercice « 37 », du chapitre « III. Utilisation de connecteurs » («III. USO DE ILATIVOS») :

37. mille reformes quils lui ont faites, la Constitution de 1980 est une merde.
A) Avec les
B) À cause des
C) Malgré les
D) Grâce aux
E) Nonobstant les. ${ }^{25}$

Malgré les divers choix proposés pour compléter le sens de la phrase (avec, à cause de, malgré, grâce à, nonobstant), le jugement de valeur qu' elle porte sur la constitution chilienne ne change pas. Celle-ci demeure " una mierda", indépendamment du choix que le lecteur adopte. L'ironie est ressentie avec cette liberté de choix qui fige le sens de la phrase dans une vision sombre du pilier de la république chilienne. Lironie répétée renforce cette vision critique de valeurs ancrées dans la société et de l'idée que l'éducation préserve la liberté, comme nous le montrent les exercices qui font référence aux universités et à leurs étudiants : «40. Les étudiant vont l'Université étudier, et non penser ${ }^{26}$. Une nouvelle fois les règles du libre choix sont transgressées par une seule possibilité de compléter : avec la préposition espagnole « $a$ ». Cette imposition du choix conduit à la formulation en français «Les étudiants vont à l'université pour étudier, et non pour penser ». Cette ironie énonce une contradiction des valeurs éducatives. Ces dernières sont plutôt en harmonie avec une société réglementée par une constitution jugée " de merde ". Ainsi l'exercice " 41 » présente des étudiants qui vont à l'université soit : pour dormir et non pour mourir ; pour boire (de l'alcool) et non pour penser; pour étudier et non pour protester; pour pleurer et non pour lire ; pour acheter et non pour faire les vitrines. L'exercice " 42 » prend la suite avec la formulation «Et s'ils ont encore de l'énergie, pour cela

24 Ibid., p. 78 : «[...] tuve que estudiar Pedagogía en Religión, ni siquiera creía tanto en Dios».

25 Ibid., p. $37: 37$. las mil reformas que le han hecho, la Constitución de 1980 es una mierda. A) Con, B) Debido a, C) A pesar de, D) Gracias a, E) No obstante. estudiar, no pensar.". 
il y a le sport " avec la préposition " pour " ("para ) comme le seul choix possible. L'exercice « 43 " clôt cette série sur les étudiants avec une combinaison de choix qui dresse un constat de dégénérescence sociale :

43. Et s'il leur reste

A) de l'espoir la réalité

B) des frustrations pour cela il y a

C) des illusions le vide

D) des pierres

la pasta de cocaïne. ${ }^{27}$

E) des neurones

la picole

Les alternatives lexicales forment un groupe sémantique évocateur d'une vision sombre de la réalité. Dans cette "realidad» qui supprime tout espoir ou qui jette les "ilusiones» dans le vide, se confrontent des solutions palliatives comme les drogues bon marché (" el copete ", "la pasta base »). Les ironies superposent au discours narratif un bilan négatif de la société, un renversement de valeurs qui accepte la révolte en tant que justification de la violence de l'État, comme le montre l'alternative D) (" et s'il leur reste des pierres, pour cela il y a la police»).

La parodie offre ainsi un champ d'action plus large à l'ironie qui s'étend à la critique d'une société compartimentée et incapable de mettre en question la ségrégation sociale. En effet, l'exercice numéro " 62 " de la partie "IV. Élimination de phrases ", souligne avec une contigüité de souvenirs anachroniques, le pouvoir de l'éducation. Les gens qui évitent de se parler et de se regarder dans un ascenseur actualisent le souvenir d'une humiliation publique subie à l'école pour avoir embrassé une fille : "Le châtiment fut de les obliger à se tenir face à face, mains dans les mains et à se regarder fixement, au milieu de la cour, toute la récréation $»^{28}$. Ainsi, Zambra relie l'intimité au plan collectif par la non-communication et la peur de l'autre. Ce narrateur, châtié dans la cour de l'école, étend à l'ensemble de la société les conséquences de sa punition : "Au Chili personne ne salue personne dans les ascenseurs. $\mathrm{Tu}$ montes et fais semblant de ne pas voir, d'être aveugle. Et si tu salues ils te regardent bizarrement, des fois ils ne te répondent même pas ${ }^{29}$. La réglementation agit contre les individus et leurs liens sociaux. Ceci est renforcé par d'autres passages ironiques du livre, comme dans l'exercice « 63 ». Un narrateur omniscient rend compte de la vie de son ami (et de la sienne) selon les préceptes d'une élite économique dans une série de douze paragraphes. Le

27 Idem. : 43. Y si les quedan para eso está A) esperanzas - la realidad, B) frustraciones - el copete, C) ilusiones - el vacío, D) piedras - la policía, E) neuronas la pasta base.

28 Ibid., p. 52 : "El castigo fue obligarlos a estar frente a frente, tomados de ambas manos y mirándose fijo, en el centro del patio, todo el recreo".

29 Ibid., p. 51 : "En Chile nadie saluda en los ascensores. Te subes y finges que no ves, que eres ciego. $Y$ si saludas te miran raro, a veces ni siquera te saludan de vuelta". 
narrateur, qui fait l'apologie de son ami, fait également un plaidoyer pour sa propre éthique de vie : "Ce qu’ils disent m'importe, me fait mal. C'est comme s'ils parlaient de moi ${ }^{30}$. Le narrateur établit alors une distinction binaire entre ceux qui parlent de son ami (les autres) et ceux qui partagent son style de vie ("nous »), distinction en accord avec la séparation des rôles sociaux inculqués à l'école avec la langue elle-même.

La non-communication et le déterminisme social qu'exerce cette éducation reviennent avec ironie dans la description d'une vie où les domestiques sont appelés avec une clochette : "Peut-on savoir quel est le problème avec la clochette ? Vaut-il mieux appeler les domestiques en hurlant ? " ${ }^{31}$. Le ton ironique et l'absurdité de la comparaison permettent de situer le locuteur dans le champ social. De même, la soumission des domestiques est présentée avec cynisme par ce narrateur qui défend les privilèges de son ami et, par extension, d'une classe. La description de la femme de ménage qui devait suivre son ami en vacances dans une station balnéaire porte le sceau du mépris social : " un mois de vacances tout payé, la vieille $»^{32}$. Ce narrateur parodie un discours identitaire qui, malgré la distinction entre " eux » et " nous » traverse avec ses valeurs l'ensemble de la société. De cette manière, les valeurs d'une économie libérale de marché se montrent sous un angle émancipateur favorable à l'échange et à la relativisation de l'histoire et des crimes commis contre l'humanité :

Maintenant il y a ceux qui disent qu'ils n'étaient pas au courant des disparitions, des tortures et des assassinats. Bien sûr qu'ils savaient. Il savait, je savais, tous nous savions. [J]e me rappelle il y a quelques années, une fois où nous étions à Rome, dans un hôtel magnifique, et un exilé nous a approchés en tenant par la main une rousse très mince. Il ne m’a pas plu l'exilé, il était lourd et grande gueule, mais mon ami s'est lié d'amitié avec lui, après ils ont même fait des affaires ensemble. ${ }^{33}$

La diversité désamorcée par un silence généralisé favorise l'homogénéité sociale, où les individus servent la suprématie de l'argent comme extension de l'identité. Cette identité est le produit d'une mémoire faussée et en même temps, elle offre l'opportunité à chaque individu de la perpétuer, dans une relation de réciprocité. Par exemple, dans l'exercice « $65 »^{34}$, où le narrateur est à la fois le chauffeur et le mémorialiste d'un homme d'origine pauvre parvenu

30 Ibid., p. 54 : «Me importa lo que dicen, me duele. Es como si hablaran de mi ».

31 Idem. : "YY cuál es el problema si se puede saber con la campanilla? ¿O sea que es mejor tratar a la empleada a grito pelado?".

32 Idem. : "Un mes de veraneo gratis, la vieja".

33 Ibid., p. 55 : "Ahora hay varios diciendo que no sabian de las desapariciones, de las torturas, de los asesinatos. Claro que sabian. El sabia, yo sabia, todos. [M]e acuerdo hace años, una vez que estabamos en Roma, en un hotel estupendo, y se nos acercó un exiliado que andaba de la mano con una colorina bien flaquita. Me cayó mal el exiliado, lo encontré denso y cachetón, pero mi compadre terminó de lo más amigo con él, después hasta hicieron negocios".

34 Ibid., p. 59. 
après avoir gagné à la loterie et farouche supporteur du dictateur Pinochet. Le récit raconte les péripéties du narrateur contraint de suivre son patron dans un voyage qui les mène du nord au sud du Chili. Dans son récit se mêlent des symboles de la culture populaire chilienne, comme une Peugeot 505, un présentateur de télévision célèbre, le dictateur, la prostitution et l'alcool. Ce long périple se solde par la publication d'un livre qui déforme les faits, volontairement édulcorés afin de satisfaire son commanditaire. L'histoire offre alors l'image d'une société corrompue. Le nouveau riche, dont la fortune tient à la chance et non à l'effort, est un homme grossier et violent qui supporte mal l'alcool. Pour sa part, le narrateur ment par pur intérêt pécuniaire en écrivant les mémoires frauduleuses de son patron.

Finalement, la soumission ou l'acceptation de l'illusion comme une identité trompeuse, sont des leitmotivs du récit : "Va te faire foutre, il m'a répondu. Je n'ai rien dit, j'étais habitué à ce genre d'humiliations " ${ }^{35}$. Avec la distance critique de l'ironie, Zambra choisit de ne pas confondre la position de l'observateur avec celle du juge et offre la symbolisation d'un statu quo dans lequel il n'y a pas d'êtres purs ou exemplaires. C'est le sens de ce "il savait, je savais, nous tous savions (à propos des tortures, assassinats et disparitions) », de l'exercice « 63 ». Peut-être, les valeurs identitaires hégémoniques mobilisées par l'éducation ne laissent-elles guère le choix d'une annihilation filiale. Ce souhait, de délivrance du passé et d'éloignement dans un présent qui serait complètement différent, représente bien l'impasse d'une conscience silencieuse, mais éveillée. Ceci s'exprime dans l'exercice " 64 ", où le narrateur emprunte la voix de Manuel Contreras, fils de Manuel Contreras, le chef de la police secrète de la dictature alors incarcéré : "Quand mon père mourra, je pourrai avoir une vie et un fils. Il sera le fils de Manuel Contreras. Mais je ne l'appellerai pas Manuel. Je dirai à la mère de lui choisir un autre prénom. Je ne veux pas être le père de Manuel Contreras ${ }^{36}$. Rompre avec la gémellité reviendrait à rompre avec l'ordre binaire.

L'autre alternative, c'est simplement la pérennité des valeurs assimilées dans une compétition pour la meilleure place que l'éducation puisse attribuer : "Précisément dans ce lycée, en théorie le plus exigeant du Chili, copier était une tâche plutôt facile [...]. Écrire n'était pas nécessaire, pas de développement, aucune idée propre ${ }^{37}$. L'ironie du renversement des valeurs liées à l'éducation, crée une complicité collective. Dans ce cas précis, c'est par la copie, la tricherie pendant les épreuves, que s'ouvre le chemin vers la recon-

35 Ibid., p. 62 : "Ándate a la concha de tu hermana, me respondió. Me quedé callado, estaba acostumbrado a esas humillaciones".

36 Ibid., p. 57 : "Cuando mi padre muera podré tener una vida y un hijo. Será el hijo de Manuel Contreras. Pero no lo llamaré Manuel. Le diré a la madre que elija otro nombre. No quiero ser el padre de Manuel Contreras".

37 Ibid., p. 71 : "Justamente en ese colegio, en teoria el más exigente de Chile, copiar resultaba más bien fácil [...]. No habia que escribir, no habia que desarrollar nada, ninguna idea propia". 
naissance d'autrui et de la communauté : " Je crois qu'avec la triche nous sommes sortis un peu de l'individualisme pour devenir une communauté $"^{38}$. L'ironie met en évidence le grotesque de l'image du langage conçu comme un piège. Les individus assimilent une identité qui accepte la soumission comme une valeur sociabilisante au prix, paradoxalement, de la négation de l'autre. Par conséquent, cette communauté est fondée dans la violence à la différence et dans l'inversion éthique de l'être pour le paraître.

\section{Conclusion}

Cette micro-fiction dans le discours expose, à notre avis, le travail essentiel de l'ironie et de la parodie par rapport aux valeurs identitaires dans Facsimil. Il s'agit d'une histoire qui montre un mécanisme de conditionnement aux valeurs porteuses d'une contradiction essentielle avec le bien-être collectif. Dans ce sens, la parodie du texte institutionnel et les moments d'ironie permettent à Zambra le signalement d'un processus identitaire qui se décompose en même temps qu'il réaffirme son hégémonie. Mais, comme nous l'avons montré, c'est une lecture de l'ensemble qui permettra d'interpréter cette "ironie du sort ", en tant que situation d'anomalie morale et éthique, de renversement de ce que le langage exemplaire prétend mobiliser comme identité. Le rôle de l'ironie est capital dans cette lecture, car elle n'est pas phrastique et confie aux lecteurs la capacité de l'identifier comme telle. Ce rôle demeure potentiel et se manifeste dans la rencontre entre le point de vue que Facsimil porte sur le monde et l'horizon d'attente du lecteur. Dans ce sens, il y a une dimension politique qui ne sera jamais close dans le roman de Zambra, car elle interpelle une diversité de sensibilités d'interprétation dont la seule constante est l'exposition d'une fatalité sociale. Le discours épisodique du roman, le récit scindé par des questions et leurs réponses, a du sens car l'ironie permet aux lecteurs d'arrêter le flux de l'allégorie du système éducatif. Grâce à elle encore, dessinant une sociabilité aliénée de l'autre la lecture critique du monde est possible. Ce livre ne propose pas une vision moralisante, et, dans cette perspective, il procède de l'ironie postmoderne qui, comme Pierre Schoentjes le note : " ne cherche plus à aller au-delà du paradoxe $»^{39}$. Elle fait le constat, avec une certaine mélancolie, d'un écart entre les valeurs humanistes et un système absurde, écart perceptible seulement si le lecteur prend la distance nécessaire pour ne pas lire ce roman comme une tragédie. Ce que l'ironie permet est alors l'actualisation à des degrés divers d'une mémoire sociale, elle permet sa survie souterraine par rapport à celle de l'ordre du système dominant. Autrement dit, elle permet la reconnaissance de ce que Gabriel Salazar distingue comme

38 Ibid., p. 72 : "Creo que gracias a la copia salimos un poco del individualismo y empezamos a convertirnos en una comunidad».

39 Pierre Schoentjes, «Ironies postmodernes», Poétique de l'ironie, Paris, Seuil, 2001, p. 287. 
l'« historicité sociale $»^{40}$ des dominés. L'ironie travaille ainsi un écart que le lecteur place entre le texte et le contexte qui le surdétermine. En effet, la narration de Facsimil est une fiction métalinguistique dès son épigraphe et c'est le contexte institutionnel éducatif qui est le référent du discours. Par l'intertextualité de la PAA, le discours est autoréférentiel, ce qui permet de saisir la force sémantique d'une codification non accordée à l'intertexte. Cette stratégie narrative utilise, en plus de la transgression des règles avec de fausses alternatives, l'indexation dialectale d'un groupe, d'une communauté ou d'une classe sociale. Le registre d'utilisation du langage formel d'une épreuve d'espagnol est traversé par d'autres registres du langage familier qui ne se prive pas d'expressions vulgaires (comme dans l'exercice 37). Les lecteurs sont capables par leur propre compétence linguistique de saisir cette stratégie comme un écart et une manifestation de l'anomalie comme symptôme de la ségrégation sociale. L'ironie rend visible l'éducation comme une institution absurde qui restreint la mobilité sociale, émousse l'expérience historique d'un peuple en lui faisant miroiter une égalité illusoire par l'accès à l'argent et au bien-être.

L'écriture, libre dans la contrainte poétique que s'impose Zambra, présente alors un langage porteur d'une division et non d'une identité collective, tel que cela s'exprime dans ce dialogue entre père et fils : "Et cependant je sens que cet abîme qui nous sépare est plus profond et irrévocable que l'abîme qui toujours sépare les enfants de leurs parents $»^{41}$.

Nicolás Folch Maass

CRICCAL

Université Sorbonne Nouvelle, Paris 3

nashfolch@hotmail.com

\begin{abstract}
Résumé
Cet article porte sur le roman Facsímil d'Alejandro Zambra, l'un des écrivains chiliens contemporains des plus remarquables selon la critique littéraire latino-américaine et européenne. Ce roman, dans lequel l'expérimentation sert l'interaction avec les lecteurs, met en avant l'inversion des valeurs par l'éducation au sein d'une société. Notre objectif sera de présenter une analyse de Facsimil en tant qu'œuvre et jeu littéraire qui dépasse le contexte chilien par l'utilisation de l'ironie comme mécanisme de relation interne au processus de lecture.
\end{abstract}

Mots-clés

Identité, Chili, littérature contemporaine, Alejandro Zambra, parodie, ironie.

40 Voir Gabriel Salazar, "La historia como ciencia popular: despertando a los weupifes ", La Historia Desde Abajo y Desde Adentro, Santiago, Taurus, 2017, p. 86.

41 Ibid., p. 97 : «Y sin embargo siento que ese abismo que nos separa es más profundo e irrevocable que el abismo que siempre separa a los hijos de sus padres". 


\section{Abstract}

This article is centered on the Facsimil novel by Alejandro Zambra, one of the most renowned contemporary writers according to Latinamerican and European literary critics. This novel, in which the experimentation interacts with the reader, highlights the inversion of values through the education within a society. Our objective will be to analyze Facsimil as an artwork and a "literary game" that goes beyond chilean context through the use of irony as a mechanism for internal relations to the reading process.

\section{Keywords}

Identity, Chile, contemporary literature, Alejandro Zambra, parody, irony. 